\title{
EINIGE WIRBELLOSE TIERGRUPPEN DES DOLLART-EMS-ESTUARIUM
}

\author{
J. H. STOCK ${ }^{\circ}$ ) und ANNA P. C. DE VOS $\dagger^{\circ}$ )
}

(mit 3 Abbildungen und 2 graphischen Darstellungen)

Im Nachfolgenden berichten wir über einige wirbellose Tiergruppen, welche in den Jahren 1953-1956 in den Bodenproben gefunden worden sind. Fräulein A. P. C. DE Vos war mit der Bearbeitung im Jahre 1954 angefangen, aber schon bald zeigte sich die Arbeit zu umfangreich und wurde Dr. J. H. STocx ein Teil der Bearbeitung übertragen. Erstgenannte Frl. DE Vos hat sich namentlich mit der Bestimmung der Polychaeta, Ostracoda, Cladocera, Harpacticoida und Calanoida beschäftigt, während letztgenannter Dr. STоск die übrigen Crustacea und übrigen Evertebraten, wie Coelenterata, Bryozoa, Echinodermata, u.s.w. vorgenommen hat. Außerdem haben Frl. DE Vos in 1954, und Dr. STock in 1956 im Dollart-EmsEstuarium außer den regulären Serienmustern ergänzende Sammlungen gemacht. Dabei wurde speziell auf die Mikrofauna geachtet.

Leider hat der unzeitige Tod von Fräulein DE Vos es für sie unmöglich gemacht selber diese Arbeit zu vollenden. Die ökologische Auswertung ihrer Daten ist ausschließlich von Dr. STock versorgt worden, und dann noch nur teilweise durchgefuhrt. Samtliche Polychaeta warten noch auf eine ökologische Durcharbeitung.

Wo in diesem Bericht über die Bodenbeschaffenheit geredet wird, sind die Namen immer im folgenden Sinne verwendet worden:

Korngröße des Sediments

$0-\quad 5 \%$ kleiner als $2 \mu$

$\quad$ Name
Sand
lehmiger Sand
sandiger Sand
Lehm
toniger Sand
Ton

$5-8 \% \quad " \quad " \quad "$

$8-15 \% \quad " \quad " \quad "$

$15-25 \% \quad " \quad " \quad "$

$25-40 \% \quad " \quad, \quad "$

$40-100 \% \quad " \quad, "$,

Wenn nötig, werden in den nachfolgenden Seiten neben den Stationsnummern auch, - zwischen Klam-

-) Zoölogisch Museum, Amsterdam. mern -, die Anzahl Exemplare an jeder Station erbeutet, aufgeführt.

\section{COELENTERATA}

HYDROZOA

Tubularia larynx Ell. \& Sol.

Nur einmal, am 5. Aug. 1954, gefunden: Paap, auf Muschelbank auf den Watten.

Cordylophora caspia (Pall.)

Nur bei Station 334a (in der Ems) gefunden.

Clava multicornis (Forskål)

Bei einer Station, 212, in der Ems querab Ditzum angetroffen.

Dynamena pumila (L.)

Zahlreich auf Fucus in den marinen und polyhalinen Teilen des Gebietes, besonders auf der Paap.

Laomedea gelatinosa (Pall.)

Wurde an 3 Stellen gefunden:

Paap, 5. Aug. 1954.

Hoogzand, 3. Aug. 1954, und

Station 221 (Mündung der Ems).

Laomedea cf. neglecta Alder

Ist zusammen mit vorgehender Art bei Station 311 gefunden.

Laomedea dichotoma (L.)

Ist bei Stat. 305 und 311, beide N. von dem Hond, angetroffen.

Laomedea longissima (Pall.)

Rottumeroog, Muschelbank auf den Watten am 12. Aug. 1954

Paap, Muschelbank auf den Watten am 5. Aug. 1954. 


\section{Ctenophora}

Beroe ovata D. Ch.

Massenhaftes Auftreten am 20. September 1956 im Plankton der Lauwers, bei Tonne Rot 8. Auch am 12. Aug. 1954 in 5 Exemplare gesammelt im Plankton, genommen bei Station 313.

\section{Pleurobrachia pileus (L.)}

Nur vereinzelt gefunden. 1 Ex. bei Stat. 243 und 1 Ex. bei Stat. 244.

\section{ECHIUROIDEA}

Von Echiurus echiurus Pall. wurde auf einer Sandplate bei Rottumeroog am 12. Aug. 19541 ausgewachsenes Exemplar erbeutet. Ein etwa $5 \mathrm{~mm}$ langes Jungtier, das an Stat. 234 (südlich von Emden) angetroffen worden ist, gehört wahrscheinlich auch zu dieser Art.

\section{SIPUNCULOIDEA}

\section{Sipunculus nudus $\mathrm{L}$.}

Ein einziges Jungtier, von etwas mehr als $8 \mathrm{~mm}$ Länge, glaube ich auf Grund seiner anatomischen Merkmale, zu S. nudus stellen zu können. Das Tier wurde gefunden bei Station 279 (Paap).

\section{ECHINODERMATA}

Im Dollart-Ems-Estuarium ist nur Asterias rubens $\mathrm{L}$. angetroffen worden. Sie lebt hier vornehmlich von Miesmuscheln.

\section{CHAETOGNATHA}

An 3 Stellen in der marin-polyhalinen Zone wurde, nur zufälligerweise weil nicht speziell auf pelagische Formen wie Chaetognathen geachtet worden ist, die Art Sagitta setosa J. Müll. angetroffen:

244 (4); 313 (1); 319 (1).

\section{BRYOZOA}

Nur 2 Bryozoa-Arten wurden im Gebiete bisher gefunden. Gewiß sind mehrere Arten da, aber bei den Sammeltätigkeiten ist nicht besonders auf Bryozoa geachtet worden.

Conopeum reticulum (L.): $242,243,484$;

Alcyonidium mamillatum Alder: 484

(det. A. W. Lacourt, Leiden).

\section{CRUSTACEA}

\section{Copepoda}

CALANOTDA

\section{Acartia bifilosa (Giesbrecht)}

Obwohl diese Art auch auf offener See gesammelt worden ist, ist sie eine typische Brackwasserbewohnerin. Sie war in der ehemaligen Zuiderzee der wichtigste Bestandteil des Planktons. Im Dollart-EmsEstuarium kommt sie nur selten vor. An zwei Stellen im Dollart und einer im südostlichen Lauwers gefunden:

Dollart: 1 Ex. 191 A; 1 Ex. 262.

S.O. Lauwers: 2 Ex. 483.

Acartia clausi (Giesbr.)

Die typische Form dieser Art lebt auf hoher See; eine morphologisch etwas abweichende Form dringt weit bis in das Brackwasser durch. Die Art war nicht selten im untersuchten Gebiete. Sie wurde an 12 Stellen im marinen Bereich der Ems (seewärts von Delfzijl), und nur an einer brackischen Stelle im Dollart gefunden:

1 Ex.: Watten vor Delfzijl, 2 Aug. 1954

1 Ex.: Paap, auf den Watten, 5 Aug. 1954

263- 1 Ex.

$280-1 \mathrm{Ex}$.

$284-3 \mathrm{Ex}$.

$293-2$ Ex.

$294-2$ Ex.

$295-1 \mathrm{Ex}$.

1 Ex. - 261 (Hoogzand, Dollart)

2 Ex. - 311 (N. von dem Hond)

2 Ex. - 316 (S. von Westerbalje)

5 Ex. - 318 (Querab Ra).

\section{Acartia discaudata (Giesbr.)}

GuRney, 1931, sagt von dieser Art: „It appears to be a species characteristic of river mouths and harbors, where the influence of fresh water is scarcely felt". Diese These stimmt wohl mit den vorliegenden Funden überein: es liegt Material vor das an 4 Stellen auf der Paap, nördlich von Delfzijl, gesammelt worden ist:

285 (1); 290 (1); 295 (1); in Torpedonetzfang, 5 Aug. 1954 (4).

\section{Acartia longiremis (Lillj.)}

Eine nordische Art, welche bei uns ihre Südgrenze erreicht. Es liegt Material vor von 4 Stationen, alle gelegen im Wattenmeer vor der Emsmündung:

1 - Rottumeroog - 12 Aug. 1954. Muschelbank auf den Watten.

1 - Rottumeroog - 12 Aug. 1954. In Tümpeln zwischen Wellenfurchen auf den Watten.

1 Ex. - 327 (Rottum).

4 Ex. (wahrscheinlich diese Art) - 137 (Schanskerdiep).

\section{Acartia tonsa Dana}

Nach Redeke, 1935, der diese Art zum ersten Mal aus den Niederlanden erwähnte, gehört sie „zu den 
häufigsten Copepoden des niederländischen mesohalinen Sommer- und Herbst-Planktons“.

Die meisten Funde im Dollart-Ems-Estuarium liegen im eigentlichen Dollart oder eben in der Ems (querab Emden), also im Mesohalinikum. Merkwürdigerweise liegen auch einige Proben aus dem marinen Teil der Ems-Mündung, unter Rottumeroog, vor. Wie auch aus der geografischen Verbreitung hervorgeht kann diese Art sowohl im mesohalinischen Gebiete als auch auf hoher See vorkommen:

Rottumeroog: 326 (2), 328 (3).

Dollart: Torpedonetzfang in einem Priel querab Stat. 434 (Kerkeriet) $(\infty)$

245 (1); 254 (3); 258 (7); 262 (3); 259-262 (Torpedonetzfang, 14) (alle: Hoogzand).

Ems, südlich von Emden: 236 (1); 240 (1).

\section{Centropages hamatus (Lillj.)}

Diese Art lebt auf hoher See, mit vollem Salzgehalt, ist aber fähig ins Brackwasser durchzudringen. Sie ist charakteristisch für die Kustengewässer und weitaus die zahlreichste Copepoden-Art des Dollart-EmsEstuariums. Obwohl sie vornehmlich außer dem marinen Bereich vorliegt, ist sie doch auch aus dem eigentlichen Ems-Flusse vor Emden, bekannt geworden.

Rottumeroog: 321 (1); 326 (3); 327 (5); 328 (4); 363 (1); Muschelbank auf den Watten, 12 Aug. 1954 (1). In Tümpeln zwischen Wellenfurchen auf den Watten, 12 Aug. 1954 (1).

Oost Friesche Gaatje: 281 (1); 302 (1); 303 (1); 304 (4).

Südost Lauwers: 483 (2).

Möwensteert: $317(\infty) ; 318$ (2).

Emshorn: 315 (4); 316 (8).

Hond: 342 (12); $311(1) ; 313(\infty)$; $314(\infty)$.

Paap: 285 (3); 289 (1); 290 (2); 291 (1); 294 (2); 295 (6); 296 (2). Außerdem in Torpedonetzfängen am 2. und 5. Aug. 1954 auf dem Watten.

Reider Plate (Dollart): 191 (1); 191 A (1); 194 (2).

Hoogzand (Dollart): 262 (1).

Ems, S. von Emden: 232 (2); 233 (2); 234 (2); 235 (1); 236 (5); 237 (1).

\section{Eurytemora affinis (Poppe)}

Typische Vertreter von E. affinis sind im Beobachtungsgebiet nur auf dem Ems-Fluß und auf der Leda, also im süßen oder leicht brackischen Bereich gefunden:

18 Ex. -213

ca. $200 \mathrm{Ex} .-214$

$\infty-218$

ca. 30 Ex. - 220 (im Plankton)
135 Ex. -332 a

130 Ex. -339

$\infty-339$ a

9 Ex. $-339 c$

1 Ex. - 339f

$\mathrm{Zu}$ der hirundoides-Form gehörendem Material wurde z.T. zusammen mit typischer affinis auf der Ems gefangen, z.T. auch in viel salzigerem ( $\alpha$-Mesohalinikum) Bereich, namentlich in dem Dollart:

Ems: 216 (ca. 50); 217 (ca. 70); 218 (ca. 200); 219 (10).

Dollart: Hoogzand, 259-262 (1).

Kerkeriet, quer von Stat. 434. Torpedonetz in einem Priel $(\infty)$.

Außerdem wurden an zahlreichen Stationen Eurytemora's erbeutet, welche nicht näher determiniert werden konnten.

Paracalanus parvus (Claus)

Eine marine Art, die in dem Wattenmeer bei der Ems-Mündung bei 9 Stationen gesammelt worden ist, und die außerdem in dem marinen und stark-polyhalinen Teil des Estuarium bis Delfzijl durchdringt. Rottum: 321 (4); 325 (5); 327 (2). Außerdem am 12. Aug. $19541 \mathrm{Ex}$. auf einer Muschelbank, und 3 Ex. in Tümpeln zwischen Wellenfurchen auf den Watten gesammelt.

Möwensteert: 318 (1).

Emshorn: 315 (2).

Oost Friesche Gaatje: 303 (1); 304 (1).

Hond: 311 (1).

Paap: 289 (2, Bestimmung nicht sicher); 293 (2); 295 (2); 296 (2).

Pseudocalanus elongatus Boeck

Pseudocalanus elongatus ist, wie die vorgehende Art, marin in seiner Verbreitung. Er kann aber bedeutend weiter als Paracalanus ins Brackwasser vordringen. Ein solches Vordringen ins Brackwasser ist aber im untersuchten Gebiete nicht beobachtet worden. Die Verbreitungen von Paracalanus und Pseudocalanus decken sich hier, insofern man aus den nicht zu zahlreichen Beobachtungen schließen kann, völlig. Auch von Pseudocalanus liegt Material vor aus der Ems-Mündung und aus dem marinen und stark-polyhalinen Bereich der Unteren-Ems, seewärts von Delfzijl.

Emshorn: 316 (1).

Hond: 306-308 (1).

Faap: 285 (1); 291 (1).

Außerdem 48 Exemplare in einer Torpedonetz-Probe gesammelt auf den Watten, nördlich von Delfzijl, am 2. Aug. 1954. 
Temora longicornis (Müll.)

Eine marine Art, welche aber in salzigere Teile des Brackwasser-Gebietes vordringen kann. Sämtliche Funde im Dollart-Ems-Estuarium entstammen, mit einer Ausnahme, dem marinen oder polyhalinen Teil der Unteren-Ems, also seewärts von Delfzijl. Der Fundort südlich von Emden gehört $\operatorname{dem} \beta$-Mesohalinikum an.

Rottum: 327 (1).

Oost Friesche Gaatje: 304 (1).

Südost Lauwers: 483 (1).

Hond: 243 (1); 311 (2); 313 (7); 314 (5).

Paap: 271 (1); 289 (1); 290 (2).

Außerdem 1 Stück in einer Torpedonetz-Probe, gesammelt auf der Paap, am 5. Aug. 1954.

Ems, südlich von Emden: 223 (1).

CXCLOPOIDA

Cyclops (Mesocyclops) leuckarti Claus

Eine Süßwasser-Art, eine Strecke stromaufwârts der

Ems gefunden:

339 (1); 339c (5); 339 e (1).

Cyclops (Acanthocyclops) vernalis Fischer

ssp. americanus Marsh

Diese Süßwasser-Art ist nur in einigen Torpedonetz-

Proben angetroffen worden, welche im süßen Teile

der Ems (stromaufwärts Hüttermoor) gefischt worden

sind:

$338 \mathrm{a}$ (1); 339c (2); 339 e (2); $339 \mathrm{f}$ (1).

Außerdem liegen von Station 337 und 339 (beide in der Ems, zwischen Boje 18 und 22) je ein Stück einer Cyclopoide vor, welche von Frl. A. P. C. DE Vos als Cyclops viridis var. pelagica de Lint bestimmt worden ist. Ob diese Varietät mit der Unterart americanus identisch ist, kann ich aus diesem kleinen Material nicht bestimmen.

Cyclops (Cyclops) vicinus Uljanin

Nur an einer Stelle, in der Ems zwischen Festenborg und Dorenborg (Stat. 339c) wurde ein einziges Ex. dieser Art, in reinem Süßwasser, gefangen mit dem Torpedonetz.

\section{Leptinogaster histrio (Pelseneer)}

Diese Copepode, welche von Pelseneer, 1929, als Strongylopleura histrio beschrieben worden ist, gehört nach Untersuchungen von BocQueT \& STOcK (1958) zur Gattung Leptinogaster. Sie lebt halbparasitisch in der Mantelhöhle der Muscheln Abra alba und Macoma balthica. Ein einziges Stück, ein Weibchen, dieser Copepode wurde an der Station 167 lose in einer Bodenprobe gefunden. Weil Abra alba dort nicht, Macoma balthica aber wohl angetroffen ist, ist es wohl sicher da $\beta$ die Copepode an letzterer Art schmarotzt hat.

\section{Macrochiron fucicolum Brady}

Neun Exemplare wurden am 12. Aug. 1954 bei Rottumeroog zwischen Fucus und Miesmuscheln auf den Watten gesammelt. Dieser Fund ist schon vor kurzem, zusammen mit nomenclatorischen und systematischen Bemerkungen, von STocx (1957) publiziert worden. Die rein marine Art ist neu für die niederländische Fauna.

\section{Paranthessius rostratus (Canu)}

Diese Cyclopoide lebt in der Mantelhöhle verschiedener Lamellibranchia. Im Dollart scheint sie wohl fast ausschließlich an Cardium edule gebunden zu sein.

Sie ist bisher nur im marinen Bereich gefunden. Auch aus dem Untersuchungsgebiet liegen nur Funde aus diesem Bereich, also aus dem Wattenmeer und Unterer Ems seewärts von Delfzijl, vor:

Rottumeroog: 371 (22); 372 (3); 374 (6); 376 (7). Auf den Watten am 12. Aug. 1954 (1) gesammelt.

Uithuizer Watten: 390 (6); 396 (3).

Paap: 291 (3); 292 (2).

Modiolicola insignis Aur.

Eine marine Art, die an den Kiemen von Miesmuscheln (Mytilus edulis) schmarotzt. Ein Exemplar wurde beobachtet bei parasitologischer Untersuchung von 12 Miesmuscheln aus dem Sparregat am 19. Sept. 1956.

\section{Mytilicola intestinalis Steuer}

Dieser Schädling aus dem Darm der Miesmuscheln wurde zusammen mit der vorgehenden Art im Sparregat gefunden. Drei der 12 untersuchten Miesmuscheln waren infektiert.

Acanthochondria depressa (T. Scott)

Zahlreich schmarotzend an den Kiemen des Buttes (Pleuronectus flesus) im Sparregat.

\section{HARPACTICOIDA}

Ameira parvula (Claus)

„Die Art kann als eine marin-meiomesohaline, in seichtem Wasser vorkommende eurytope Art charakterisiert werden" (LANG, 1948). Ein Fund aus dem marinen Gebiet:

Rottumeroog: 2 Ex. zwischen Fucus auf den Watten. 12 Aug. 1954.

Asellopsis intermedia (T. Scott)

Diese Copepode lebt vornehmlich auf Sand und 
Schlamm. Sie wurde an 3 Stellen gesammelt, 2 im marinen, 1 im stark polyhalinen Bereich.

Rottumeroog: 6 Ex. in Tümpeln zwischen Wellenfurchen auf den Watten, am 12. Aug. 1954.

2 Ex. zwischen Fucus auf einer Muschelbank, am 12. Aug. 1954.

Paap: 2 Ex. auf den Watten (Torpedonetzfang, 5 Aug. 1954).

Canuella furcigera G. O. Sars

Im Grenzgebiet der marinen Zone bei 4 Stationen auf Sandboden gefunden.

Uithuizer Watten: 393 (1); 394 (1); 396 (1); 401 (2).

Canuella perplexa

Es liegen von dieser Art zahlreiche Funde vor aus dem polyhalinen und marinen Gebiet des Estuarium, wo sie auf Sandboden vorkommen. Canuella $p$. ist im hohen Grade euryhalin: man hat sie in Süßwasser und in Salzlagunen gefunden.

Rottumeroog: 363 (1); 367 (1).

$2 \mathrm{Ex}$. in Tümpeln zwischen Wellenfurchen, und

2 Ex. zwischen Fucus in den Watten (12 Aug. 1954).

Möwensteert: 318 (1).

Paap, und Watten nördlich von Delfzijl: 2 Ex. in Torpedonetzfängen am 2. Aug. 1954, ca. $200 \mathrm{Ex}$. am 5. Aug. 1954.

Ectinosoma curticorne Boeck

$\mathrm{Zu}$ dieser sehr euryhalinen Art sind vielleicht einige Copepoden zu bringen, eine aus dem polyhalinen Bereich, drei weitere aus dem mesohalinen Bereich: Hond: 244 (2); 306-308 (1).

Ems, sïdlich von Emden: 222 (3).

Die Bodenbeschaffenheit an den Stationen 306-308 ist Lehm bis tonigen Lehm.

Ectinosoma elongatum Sars

In der marin-polyhalinen und $\beta$-mesohalinen Zone des Dollart-Ems-Estuariums ist diese Art mehrmals gefunden:

Uithuizer Watten: 379 (1) (auf Sand).

Paap: 244 (5); in einem Torpedonetzfang am 5. Aug. 1954 (8).

Hoogzand: in einem Torpedonetzfang am 3. Aug. $1954(>80)$.

Ectinosoma gothiceps Giesbr.

Diese Meeresart trat nur in einer Probe auf, das aus der polyhalinen Zone stammt:

Hund: 306/308 (1) (auf Lehm bis tonigem Lehm).
Ectinosoma herdmani T. et A. Scott

Einmal ist $1 \mathrm{Ex}$. gefunden:

Rottumeroog: in Tümpeln zwischen Wellenfurchen auf den Watten, 12 Aug. 1954.

Ectinosoma melaniceps Boeck

LANG, 1948, hält diese Art für marin und polyhalin. Im betrachteten Gebiet trat sie nur an einer Stelle, an der Sandplate Paap, im polyhalinen Bereich, auf. Zwei Exemplare in einem Torpedonetzfang am 5. Aug. 1954.

\section{Euterpina acutifrons (Dana)}

Eine Planktonform, welche ich aber an den Sandstranden der holländischen Küste mehrmals im Sand gefunden habe. Auch aus dem Untersuchungsgebiet liegt sie aus mehreren Bodenproben vor; merkwürdigerweise nicht nur aus dem marinen und polyhalinen Gebiete, sondern auch aus der mesohalinen Zone (Ems bei Logum):

Rottumeroog: 321 (1); 327 (2); 328 (1).

Paap: 283 (2); 289 (1); 295 (1); 301 (1); 304 (2).

Ems, querab Logum: 241 (1).

So weit analysiert, ist die Bodenbeschaffenheit an dieser Station meistens Sand, bisweilen lehmiger Sand oder Lehm.

\section{Harpacticus flexus Brady et Robertson}

Diese vorwiegend marine Art trat nur in dem marinpolyhalinen Teil des Estuariums auf:

Rottumeroog: In Tümpeln zwischen Wellenfurchen auf den Watten, 12 Aug. 1954 (52). Zwischen Fucus auf Muschelbank, 2 Aug. 1954 (4).

Paap: Im Torpedonetzfang auf den Watten, 5 Aug. 1954 (10).

\section{Harpacticus gracilis Claus}

Die Art ist vorwiegend marin. Von den nachfolgenden Fundorten sind zwei in der marinen Zone, zwei andere in der polyhalinen Zone.

Rottumeroog: Zwischen Fucus auf den Watten, 12 Aug. 1954 (> 150).

Uithuizer Watten: auf Zostera, Station 394a (11).

Paap: auf Muschelbank, 5 Aug. 1954 (1).

Watten zwischen Delfzijl und Oterdum: $352(\infty)$.

Héterolaophonte minuta (Boeck)

Ein Stück aus dem Wattenmeer:

Rottumeroog: zwischen Fucus auf den Watten, 12 Aug. 1954.

\section{Longipedia coronata Claus}

Eine Meeresart, die nur im Polyhalinikum noch vor 
dringen kann. Sie wurde an einer Stelle, nördlich von der Hond Plate (Stat. 311) bei einem Salzgehalt von etwa $30 \%$ in 2 Ex. erbeutet.

Longipedia minor T. et A. Scott

Obwohl die Art als euryhalin angeschrieben steht, ist sie im untersuchten Gebiete nur an 2 marinen Fundorten gefunden:

Rottumeroog: 321 (1) auf Sand; zwischen Fucus auf den Watten, 12 Aug. 1954 (26).

\section{Microarthridion littorale (Poppe)}

Eine euryhaline Brackwasser Art, bisweilen im SüBwasser gefunden. Auch im untersuchten Gebiet kam sie bei sehr wechselnden Salzgehalten vor: in der polyhalinen Zone (Hond, Paap), in der mesohalinen Zone (Ems von Emden bis Ditzum), und im reinen Süßwasser (Ems oberhalb der Leda):

Paap: 243 (1); auch in einem Torpedonetzfang auf den Watten am 5. Aug. 1954 (9).

Hond: 306-308 (4).

Ems: 222 (39); 233 (1) (beide südlich von Emden); 212 (1); 332a (2) (beide querab Ditzum); 339e (zwischen Hilkenborg und Mark) (2).

Nannopus palustris Brady

Eine Brackwasserart, die bisweilen bis ins süße, bisweilen bis ins Meereswasser vordringt. Sie lebt vornehmlich auf schwarzem Schlick. Im Gebiete zahlreich angetroffen, und zwar fast ausschließlich im mesohalinen Brackwassergebiet.

Dollart: 183 A (3); 184 (5); 190 (1); 195 (3) (Kerkeriet).

206 (4) (Noordwal).

137 (1) (Maan Plate).

423 (1) (Hering Plate).

463 (11) (Moe Plate).

Ems: 241 (1) (querab Logum).

213 (1) (querab Ditzum).

232 (2); 233 (31); 235 (2); 240 (1) (südlich von Emden).

Außerdem 1 Ex. in einem Torpedonetzfang auf den Watten, nördlich von Delfzijl (Polyhalinikum!) am 2. Aug. 1954. Und 95 Ex. aus der Lauwersee, bei Zoutkamp, am 10. Aug. 1954.

\section{Paronychocamptus curticaudatus (Boeck)}

Trat in 2 Proben, gesammelt im polyhalinen Gebiet, auf:

Watten nördlich von Delfzijl: In einem Torpedonetzfang am 2. Aug. 1954 (2).

Hond: 306/308 (1) (auf Lehm bis tonigem Lehm).

\section{Paronychocamptus nanus (Sars)}

Eine Brackwasserart, die nicht im eigentlichen Meer vorkommt. Sie wurde an zwei Stellen in der Ems, südlich von Emden (also in der mesohalinen Zone) erbeutet:

222 (1); 233, in einem Priel (5).

\section{Platychelipus littoralis Brady}

Eine Brackwasserform die vor Allem auf schlammigem Boden lebt. Im Dollart-Ems-Gebiet ist sie vornehmlich in den von Süßwasser beeinflußten Gebieten (Ems, Dollart) gefunden, und nur vereinzelt in der polyhalinen und marinen Zone.

Rottumeroog: 326 (3); 327 (1).

Hond: 306-308 (37).

Dollart: 183A (8); 183 (6); $184(26) ; 185(4) ; 186(1)$; 187 (2); 190 (1); 192 (2); 194 (1); 195 (7); 201 (2); 206 (4) (= Kerkeriet/Noordwal); 463 (1) (= Moe Plate).

Ems: 232 (3); 233 (21); 235 (1); 238 (1); 239 (1) (südlich von Emden).

214 (1) (querab Hatzum).

215 (1) (querab Kritzum).

217 (1) (querab Jemgum).

An 16 dieser Stationen wurde die Bodenbeschaffenheit analysiert. $P$. littoralis wurde an 3 dieser auf Sand, an 1 auf lehmigen Sand, an 8 auf sandigen Lehm, und an 4 auf Lehm festgestellt.

\section{Pseudobradya beduina Monard}

Eine südliche Meeresart, beschrieben aus Tunesien und Algerien.

31 Ex. Rottumeroog, in Tümpeln zwischen Wellenfurchen auf den Watten, 12 Aug. 1954; ibidem, zwischen Fucus (30 Ex.) (marines Bereich).

1 Ex., 244, zwischen Hond und Paap (polyhalines Bereich).

Paap, in Torpedonetzfangen am 5. Aug. 1954 (7).

\section{Pseudobradya minor (T. et A. Scott)}

Eine euryhaline Art, welche mehrmals auf Sand oder schlammigem Sand gefunden worden ist. Sie ist nur in 4 Exemplaren vertreten, gefunden auf den Watten in der Ems, südlich von Emden (Stat. 222), also im Mesohalinikum.

\section{Stenhelia palustris Brady}

Eine oft auf Sand und Schlamm gefundene marin bis $\beta$-mesohaline Art. Im Untersuchungsgebiete dringt sie ziemlich weit ins Mesohalinikum vor, und ist in der Ems, sowie in den wenig salzigen Teilen des Dollarts gefunden. Sie liegt aber auch aus dem Polyhalinikum vor (Hond). 
Hond: 306-308 (4) (auf Lehm).

Dollart: 463 (1) (= Moe Plate) (auf Lehm).

Ems, südlich von Emden: 233 (17) (auf Sand).

Tachidius discipes Giesbr.

Eine der Kennarten für das Mesohalinikum (REDEKE), die jedoch gelegentlich auch wohl bei höherem oder niedrigerem Salzgehalt angetroffen worden ist.

Die meisten vorliegenden Funde stammen aus dem meso- und oligohalinen Bereich der Ems und Dollart. Eine Probe aber entstammt der marinen Zone (394a), zwei der Süßwasserzone der Ems (339c und $339 \mathrm{~d}$ ).

Uithuizer Watten: 394 a (2), auf Zostera.

Dollart (Hoogzand): 262 (1); überdem 1 Stück in einem Torpedonetzfang an gleicher Stelle, 3 Aug. 1954.

Ems: 216 (1) (querab Midlum).

337 (1) (querab Kleihusen).

217 (1) (querab Jemgum).

339 (8) (querab Bentersiel).

218 (7), 339a (ca. 20) (querab Spitland).

339 c (1) (querab Ferstenborg).

339d (2) (bei Eisenbahnbrücke Weener).

Thompsonula hyaenae (Thomps.)

Da diese nur, und in nicht unbedeutender Menge, in Torpedonetzfangen vertreten ist, und nicht in den normalen Bodenproben, scheint sie weniger an dem Boden gebunden zu sein als die meisten anderen Arten. Sie ist nur in der polyhalinen und marinen Zone beobachtet worden:

Rottumeroog: In Tümpeln zwischen Wellenfurchen auf den Watten, 12 Aug. 1954 (9). Zwischen Fucus, 12 Aug. 1954 (9).

S.O. Lauwers: 483 (2), Torpedonetzfang.

Paap: 8 Ex. am 2. Aug. 1954, 63 Ex. am 5. Aug. 1954, in Torpedonetzausbeuten.

Tisbe furcata (Baird)

Eine Meeresart, die in der polyhalinen Zone vordringen kann. Sie ist bei Rottumeroog, zwischen Fucus auf den Watten angetroffen in 2 Ex. am 12. Aug. 1954 (marin), und weiter in 1 Ex. an der Station 311 (Hond), bei einem Salzgehalt von $30 \%$, also auf der Grenze der marinen und polyhalinen Zone.

CALIGOIDA

Lepeophtheirus pectoralis (O.F.M.)

Sehr zahlreich auf der Haut und den Flossen des Buttes (Pleuronectus flesus) im Sparregat (19 Sept. 1956).
Lernaeocera branchialis (L.)

Einige Larven, im Copepodid-Stadium, wurden an den Kiemen von Pleuronectus flesus im Sparregat gefunden (19. Sept. 1956).

\section{Gattung Sphaeronella Sel.}

Mehrere Arten dieser Gattung, die noch nicht aus unserem Faunagebiete bekannt waren, wurden angetroffen.

Schmarotzend an Corophium arenarium Crawford wurde eine bedeutende Anzahl Sphaeronellen gefunden. Die Pupae fanden sich häufig an den Beinen, Marsupialplatten und Pleopoden gehaftet. Ausgewachsene Männchen, Weibchen und deren Eiballen wurden, obwohl viel seltener als die Pupae, auch gefunden, und zwar immer im Marsupium. Die wenigen erwachsenen Weibchen zerfallen in 2 recht verschiedenen Typen, die ich hier als zwei selbständige Arten aufführe. Es ist mir nicht gelungen auch 2 Typen in den Männchen anzutreffen. Es ist entweder möglich, daß nur Männchen gehörend zu einer der weiblichen Typen gefunden worden sind, oder daß die Männchen der beiden Arten nicht oder kaum verschieden sind (was in der Arten-Gruppe wozu die Weibchen gehören, die S. leuckarti-Gruppe, mehrmals der Fall scheint zu sein, allerdings nach HanseN, 1897).

Sphaeronella devosae Stock (nov. spec.) (Fig. 1)

Weibchen: Gesamtlänge $956 \mu$ (Exemplar von Stat. 410). Länge des Kopfes $214 \mu$. Breite des Kopfes $304 \mu$. Größte Breite des Körpers $653 \mu$. Der Kopf macht also etwa $22 \%$ der Gesamtlänge aus, die Breite des Kopfes etwa $47 \%$ der größten Breite.

Der Körper ist fast gänzlich mit Schuppen besetzt; solche Schuppenartigen Bildungen sind sonst nur bei Sph. vestita bekannt. Eine Genitalregion ist kaum abgegrenzt. Nur an der proximalen Seite des Rezeptaculums seminis findet man eine undeutliche Andeutung des Randes der Genitalregion. Die beiden Rezeptacula stoßen in der Median-Linie zusammen. Furcalzweigen ziemlich dicht nebeneinander. Zwischen den Furca und den Genitalöffnungen findet man zahlreiche lange, nicht zu Schuppen umgebildete Haare.

Die ersten Antennen sind viergliedrig. Die zweiten Antennen eingliedrig. An der ventralen Seite des Kopfes gibt es 5 Gruppen Haare: (a) an der Außenseite der Basis des 1. Mx.; (b) lange Haare an der fronto-medialen Seite des 2. Mx., bei dessen Basis; (c) eine kleine Gruppe seitlich der Einpflanzung des 2. Mx.; (d) eine Gruppe vor und (e) eine Gruppe 


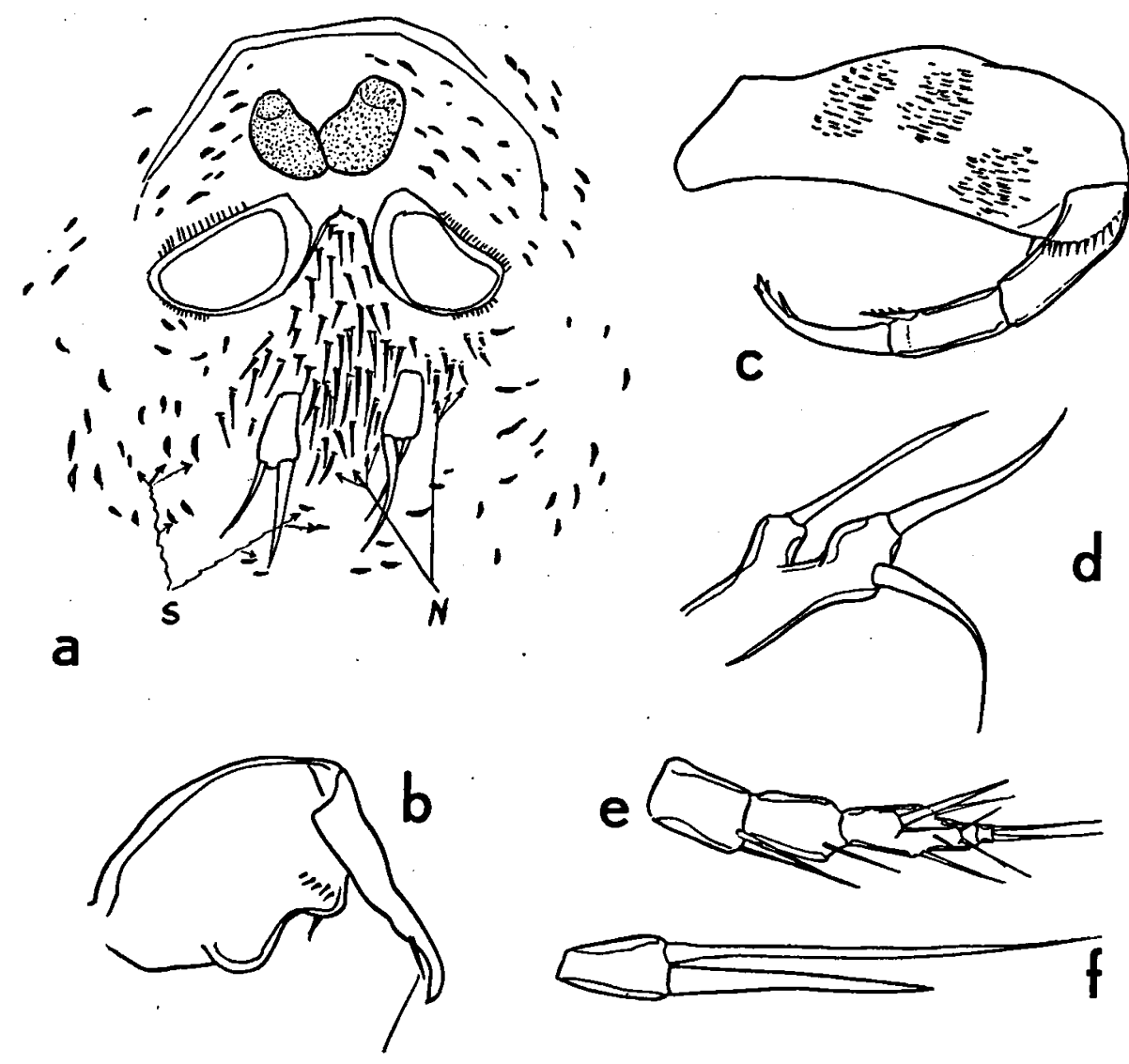

Fig. 1. - Sphaeronella devosae nov. spec., ㅇ․

a, Genital-Region ( $\mathrm{S}=$ Schuppenartige Haare; $\mathrm{N}=$ normale, nicht umgebildete, Haare); b, 2. Maxille; c, Maxilliped; d, 1. Maxille; e, 1. Antenne; f, 2. Bein.

hinter der Einpflanzung der Mxp. Die 2. Mx. tragen 2 Schwellungen am Basalglied. Die Kralle trägt an der Innenseite auf etwa $3 / 4$ seiner Länge eine Dorne. Männchen: Sehe weiter unter Sphaeronella spec. Vorkommen: Nur die erwachsenen Weibchen dieser Art können von der nächsten Art unterschieden werden. Solche erwachsenen Weibchen wurden an der Station 400 und 410 (je ein Stück) im Marsupium von Cor. arenarium gefunden. Diese Art sei Fräulein A. P. C. DE Vos, Conservatrix am Zoologischen Museum Amsterdam, gewidmet, zu Ehren ihrer vielen Beiträge zur Kenntnis der niederländischen Fauna, und insbesondere auch zu der biologischen Forschung im Dollart-Ems-Estuarium. Das Material ist im Zoologischen Museum Amsterdam, mit Kat.nr. 100.430431.

Bemerkungen: Diese Art gehört zu der leuckartiGruppe, wie von Hansen, 1897, definiert. Sie unterscheidet sich von allen Arten dieser Gruppe durch eine Kombination von Merkmalen, die Schuppen- artige Bewehrung des Körpers, die reduzierte Ausbildung der Genitalregion, die langen Haare zwischen Furca und Genital-öffnungen, und die relativen Abrisessungen des Körpers.

Sphaeronella ecaudata Stock (nov. spec.) (Fig. 2)

Weibchen: Nur wenig kleiner als die vorgehende Art. Gesamtlänge des Körpers $854 \mu$; größte Breite $529 \mu$; Länge des Kopfes $169 \mu$, größte Breite des Kopfes $248 \mu$. Auch die Längen-Breiten-Verhältnisse des Kopfes und des Körpers sind der vorgehenden Art völlig gleich.

S. ecaudata unterscheidet sich aber wesentlich in den nachfolgenden Merkmalen: Der Körper ist fast nackt. Nur an der distalen Seite der weiblichen Geschlechtsöffnungen findet man einige kurze, nicht schuppenartig ausgebildete Haare. Die beiden Rezeptacula seminis sind getrennt in der Medianlinie. Die ganze Genitalregion ist gut ausgebildet. Sie hat die Form einer gut chitinisierter, breit-birnförmiger Platte. Die 

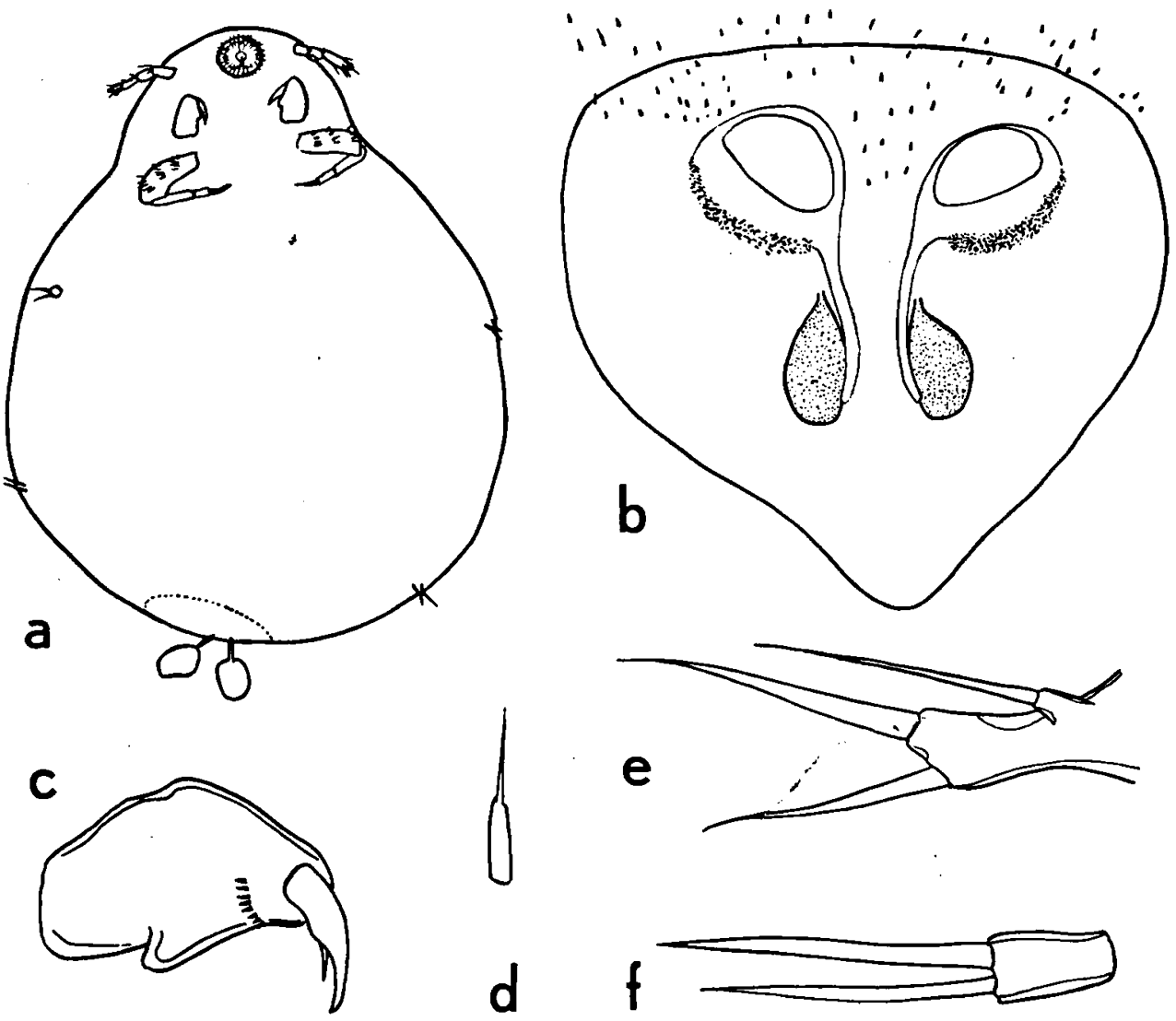

Fig. 2. - Sphaeronella ecaudata nov. spec., ㅇ.

a, Das ganze Tier, von der Bauchseite; b, Genital-Region; c, 2. Maxille; d. 2. Antenne; e, 1. Maxille; f, 2. Bein.

Furca fehlt fast völlig, nur eine kleine Erhöhung deutet auf das Furca-Rudiment.

Antennen, 1. Mx. und Mxp. fast vollständig identisch mit den gleichen Teilen von S. devosae. 2. Mx. nur mit einer Schwellung am Basalglied. Die Kralle trägt an der Innenseite eine Dorne auf etwa die Hälfte ihrer Länge. Die Behaarung der ventralen Seite des Kopfes ist auf 3 Gruppen beschränkt: (a) eine Gruppe an der Außenseite der Einpflanzung der 1. Mx.; (b) eine Gruppe an der Innenseite der 2. Mx., bei dessen Basis; (c) eine kleine Gruppe kurzerer Haare fronto-lateral der Einpflanzung der Mxp.

Vorkommen: Nur 1 ausgewachsenes Weibchen, in dem Marsupium einer Corophium arenarium gesammelt an Stat. 401 (Zoolog. Museum, Amsterdam, Kat.nr. 100.429).

Bemerkungen: Das Fehlen einer wohlentwickelten Furca, der nackte Körper, die Ausbildung der Genitalregion und die relativen Abmessungen des Körpers kennzeichnen diese Art genügend.
Sphaeronella spec. (Fig. 3)

Unter dieser Bezeichnung führe ich die Männchen, Pupae und Eiballen auf, welche an Corophium arenarium gefunden wurden und wovon es nicht klar ist, ob sie zu Sphaeronella devosae oder zu S. ecaudata gehören.

Männchen: Länge 270-281 $\mu$, größte Breite 191$208 \mu$ (Stücke von Stat. 399). Stirnrand 3-lappig. Mittellappen etwas breiter als lang; Seitenlappen etwa $2 / 5$ der Länge der Mittellappen. Die Extremitäten sind in der für die Arten der leuckarti-Gruppe normalen Weise ausgebildet worden. Die Furcaläste zeigen am distalen Ende eine deutliche 2-Lappigkeit. Der Außenlappen trägt 2 Borsten, der Innenlappen 1 starke Stachel und 4-5 Borsten. In ihrem Bau scheint die Furca sich von allen bisher beschriebenen Arten der leuckarti-Gruppe (so weit es aus oft in sehr kleinem Maßstabe ausgeführten Figuren zu beurteilen ist) zu unterscheiden. Im allgemeinen ist jedoch das Männchen dem Männchen von S. chinensis sehr ähnlich (Hansen, 1897, Taf. III, Fig. 1). 


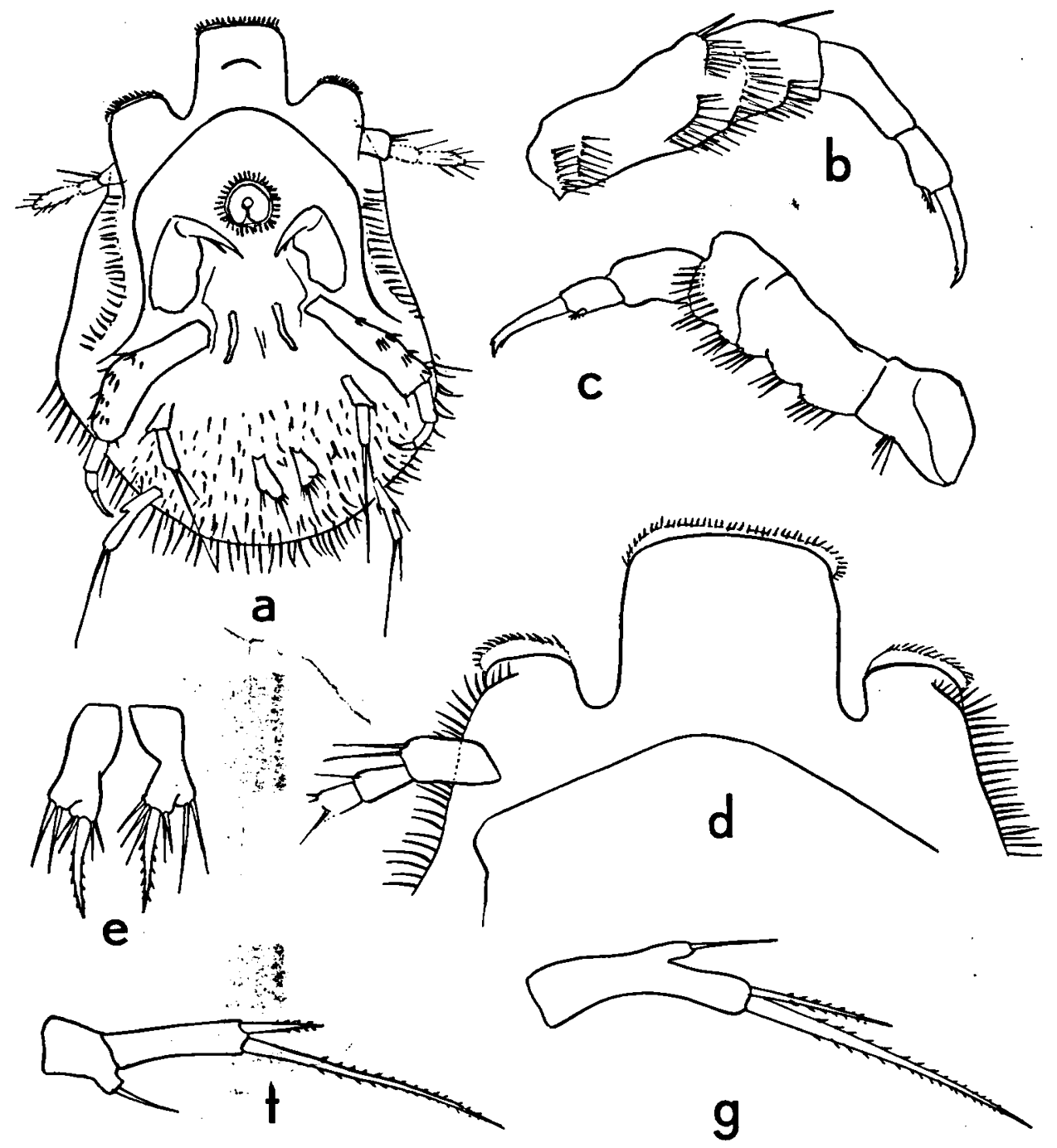

Fig. 3. - Sphaeronella spec., ồ ô.

a, $\delta$ von Stat. 399, von der Bauchseite; b, Maxilliped (Stat. 401); c, Maxilliped (Stat. 400); d, Kopfrand (Stat. 400); e, Furca (Stat. 401); f, 1. Bein (Stat. 401); g, 2. Bein (Stat. 400).

Eibälle: Drei gemessene Eibälle (Station 401) sind $505 \times 518 \mu, 619 \times 484 \mu$ und $474 \times 439 \mu$.

Pupae: Sind an verschiedenen Stationen recht zahlreich an den Beinen, Marsupialplatten, Kiemen und Pleopoden von Corophium. Die Abmessungen der Pupae wechseln stark: ich habe Pupae gesehen von $180 \times 135 \mu$, aber auch von $325 \times 280 \mu$.

Vorkommen: 1954, Stat. 174: 7 Eibälle in einem Weibchen von Cor. arenarium;

1956, Stat. 399: 3 Männchen, mehrere Pupae und Eibälle in Cor. ar.

1956, Stat. 394: 1 Pupa in Cor. ar.
1956, Stat. 400: 2 Männchen, 5 Eibälle an Cor. ar. 1956, Stat. 401: 1 Männchen, mehrerer Pupae und Eibälle an Cor. ar.

1956, Stat. 410: 2 Pupae an Cor. ar.

Sphaeronella paradoxa Hansen

Mehrere Exemplare dieser Art, die neu ist für die niederländische Fauna, wurden im Marsupium der Amphipode Bathyporeia pelagica gefunden (Station 319).

\section{Cladocera}

Alle bei den Untersuchungen beobachteten Clado- 
ceren sind Süßwasserarten. Eine Anzahl dieser Arten dringt aber in die oligohaline Zone vor.

In der Ems nur im Süßwasser beobachtete Formen sind:

Bosmina coregoni f. typica Baird

339 (1); 339 a (1); 339b (2); 339c (3); $339 \mathrm{~g}$ (2).

Bosmina longirostris $\mathrm{f}$. brevicornis Hellich $339 \mathrm{~b}$ (1).

Bosmina longirostris f. similis Lillj. 339 a (1).

Bosmina longirostris f. typica (O.F.M.) $339 \mathrm{~b}$ (3); $339 \mathrm{e} \mathrm{(1).}$

Ceriodaphnia quadrangula (O.F.M.) $339 \mathrm{e}(1)$.

Daphnia longispina (O.F.M.) $339 \mathrm{~g}(1)$.

Daphnia longispina f. cucullata Sars 339 a (1); 339b (1).

Daphnia pulex (de Geer) 339 b (2).

Eurycercus lamellatus (O.F.M.) 339 e (1).

Simocephalus vetulus (O.F.M.) 339 c (1); 339h (1).

In der oligohalinen Brackwasserzone (bsw. im Brackwasser und im Süßwasser) kommen vor:

Alona affinis (Leydig) 213 (1); 214 (1).

Alona quadrangularis (O.F.M.) 332 a (1); 339 a (2); 339b (2).

Bosmina coregoni Baird 214 (1); 218 (2); 332 a (1).

Bosmina longirostris cornuta Jurine 214 (1); 339a (2).

Chydorus sphaericus O.F.M. 332 a (1); 339 a (3).

Iliocryptus sordidus (Lieven) 213 (1).

\section{Amphipoda}

Familie Haustoriidae

Drei Gattungen dieser Familie sind im Gebiete vertreten. Da sie alle eine gleiche Lebensweise haben, nämlich eingegraben in Sand, und nicht zu Lehmigen Sand, werden sie hier zusammen betrachtet.

Folgende Arten kommen im Dollart-Ems-Estuarium vor:

Bathyporeia pilosa Lindström

Bathyporeia sarsi Watkin

Bathyporeia elegans Watkin

Bathyporeia pelagica Bate

Haustorius arenarius (Slabber)

Urothoe grimaldii Chevreux var. inermis.
In der nachfolgenden Tabelle sind die Stationen an welchen diese Arten gefunden worden sind, nebst der petrographischen Zusammenstellung des Bodens dieser Stationen zusammengefaßt.

In der Tabelle stehen die marinen Fundorte oben; je niedriger der Salzgehalt, je niedriger auch die Stellung in der Tabelle.

Es ist klar, daß alle Arten der Haustoriidae sich nur in grobkörnigen Böden einbohren. Wenn ca. 70 bis $80 \%$ aus Körnchen größer als $105 \mu$ besteht, scheint der Boden den Haustoriiden wohl die besten Lebensbedingungen zu bieten.

Es ist auch wohl deutlich, daß Urothoe grimaldii var. inermis nur in den marinen Teilen vorkommt, und speziell dort wo die Korngröße für Bathyporeia ungünstiger wird, d.h. wo mehr als ca. $35 \%$ aus Körnchen unter $105 \mu$ besteht. Die vorherrschende Art in dem marinen Teil des Estuarium ist ohne Zweifel Bathyporeia sarsi. In den mesohalinen Teilen des Gebietes tritt sie nur noch vereinzelt auf und wird völlig ersetzt durch Bathyporeia pilosa. $\mathrm{Zu}$ sammen mit pilosa, aber viel weniger zahlreich, tritt auch $B$. elegans auf; zusammen mit sarsi (aber recht selten im Gebiete) findet man B. pelagica. B. pilosa und sein Begleiter B. elegans sind die einzigen Arten der Familie die in die Ems durchdringen.

Familie Atylidae

Nototropis swammerdami (M-Edw.)

Diese marine Amphipode kann ziemlich große Versüßung ertragen. Sie ist an zwei Stationen erbeutet worden:

Ra: im Oberflächenplankton, am Abend auf Licht,

20 September $1956(\infty)$.

Kerkeriet: Station 434 (1).

Familie Gammaridae

Die Systematik dieser Familie hat sich in den letzten 10 Jahren sprunghaft entwickelt. Wo man in den Niederlanden, noch nach 1945 nur 5 GammarusArten anerkannte (locusta, marinus, duebeni, pulex, roeseli), so ist die wirkliche Anzahl nach meiner Überzeugung ca. 2 bis $3 \mathrm{Mal}$ so viel. In dieser Arbeit kann ich zwei weitere für das niederländische Faunengebiet neue (d.h., nie früher in der Literatur aus den Niederlanden erwähnt gewordene) Arten aufführen, nämlich Gammarus salinus Spooner und G. oceanicus Segerstråle.

Gammarus zaddachi Sexton

Diese, vornehmlich im Mesohalinikum sehr verbreitete Art, tritt nur in einer Probe auf: 
Tabelle I

Verbreitung der Haustoriidae im Gebiete

\begin{tabular}{|c|c|c|c|c|c|c|c|c|c|c|}
\hline \multirow[b]{2}{*}{ Station } & \multirow[b]{2}{*}{$\begin{array}{c}\text { Allgemeine } \\
\text { Ortsandeutung }\end{array}$} & \multicolumn{6}{|c|}{ Anzahl } & \multicolumn{2}{|c|}{ Kom-Größe } & \multirow[b]{2}{*}{$\begin{array}{l}\text { Name des } \\
\text { Sediments }\end{array}$} \\
\hline & & $\begin{array}{l}\stackrel{8}{0} \\
\stackrel{0}{0} \\
\text { ஜ }\end{array}$ & D & 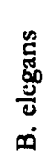 & 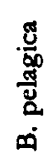 & 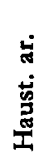 & 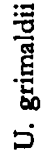 & $\%<105 \mu$ & $\%>105 \mu$ & \\
\hline$\left\{\begin{array}{l}324 \\
356 \\
357 \\
358 \\
359 \\
360 \\
361 \\
363 \\
364 \\
365 \\
366 \\
367 \\
z \\
368 \\
370 \\
319 \\
315 \\
383 \\
385 \\
387 \\
397 \\
403 \\
406\end{array}\right.$ & $\begin{array}{c}\text { Rottumeroog } \\
\text { ", } \\
" \\
" \\
" \\
" \\
\text { " } \\
\text { " } \\
\text { " } \\
\text { " } \\
\text { Querab Sparregat } \\
\text { S. v. Emshorn } \\
\text { Uithuizer Watten } \\
\text { " } \\
\text { " } \\
\text { " } \\
\text { " } \\
\text { " }\end{array}$ & 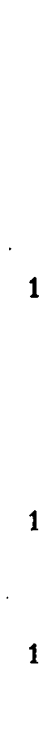 & $\begin{array}{r}2 \\
20 \\
38 \\
43 \\
4 \\
1 \\
18 \\
18 \\
70 \\
60 \\
27 \\
5\end{array}$ & & 23 & 5 & $\begin{array}{l}1 \\
1 \\
1 \\
1 \\
\\
1 \\
1 \\
1 \\
1 \\
1\end{array}$ & $\begin{array}{c}-\overline{25,7} \\
25,4 \\
21,3 \\
21,1 \\
18,5 \\
33,1 \\
28,4 \\
23,5 \\
20,4 \\
18,4 \\
28,2 \\
35,7 \\
47,9 \\
- \\
-5 \\
51,6 \\
46,2 \\
46,6 \\
60,3 \\
51,2 \\
60,5\end{array}$ & $\begin{array}{r}- \\
74,3 \\
74,6 \\
78,7 \\
78,9 \\
81,5 \\
66,9 \\
71,6 \\
76,5 \\
79,6 \\
81,6 \\
71,8 \\
64,3 \\
52,1 \\
- \\
- \\
48,4 \\
53,8 \\
53,4 \\
39,7 \\
48,8 \\
39,5\end{array}$ & $\begin{array}{c}- \\
\text { Sand } \\
" \\
" \\
" \\
" \\
" \\
" \\
" \\
" \\
\text { " } \\
\text { Sand } \\
\text { " } \\
\text { " } \\
\text { " } \\
\text { " }\end{array}$ \\
\hline 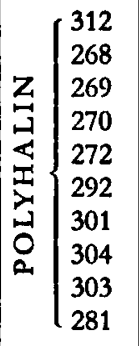 & $\begin{array}{c}\text { Hond } \\
\text { Paap } \\
" \\
", \\
", \\
" \\
\left\{\begin{array}{c}\text { Oost Friesche } \\
\text { Gaatie }\end{array}\right.\end{array}$ & $\begin{array}{l}5 \\
2\end{array}$ & $\begin{array}{l}2 \\
1 \\
4 \\
3 \\
2 \\
1 \\
1\end{array}$ & $\begin{array}{l}3 \\
1\end{array}$ & & 1 & & $\begin{array}{l}\overline{31,5} \\
- \\
24,2 \\
24,1 \\
- \\
34,0 \\
- \\
- \\
-\end{array}$ & $\begin{array}{c}-\overline{68,5} \\
- \\
75,8 \\
75,9 \\
- \\
66,0 \\
- \\
-\end{array}$ & $\begin{array}{c}\text { Sand } \\
\text { Sand } \\
\text { - } \\
\text { Lehm. Sand } \\
\text { - } \\
\text { - }\end{array}$ \\
\hline 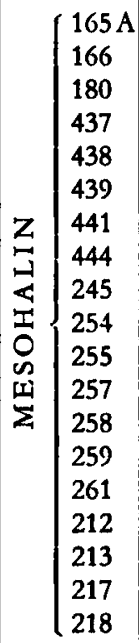 & $\begin{array}{c}\text { Dollart } \\
\text { (Reiderplate - } \\
\text { Kerkeriet) } \\
" \\
" \\
" \\
" \\
\text { Dollart (Hoogzand) } \\
" \\
" \\
" \\
\text { " } \\
\text { " } \\
\text { Ems, querab Ditzum } \\
\text { " } \\
\text { Ems, querab Jemgum } \\
\text { Ems, querab Spitland }\end{array}$ & $\begin{array}{r}10 \\
7 \\
6 \\
3 \\
16 \\
9 \\
5 \\
4 \\
1 \\
5 \\
7 \\
1 \\
9 \\
1 \\
\\
1 \\
1 \\
3 \\
3\end{array}$ & 1 & $\begin{array}{l}1 \\
3\end{array}$ & & & & $\begin{array}{l}- \\
36,3 \\
20,3 \\
29,5 \\
21,6 \\
26,5 \\
37,3 \\
48,8 \\
24,8 \\
14,1 \\
- \\
- \\
- \\
- \\
- \\
- \\
-\end{array}$ & $\begin{array}{l}- \\
63,7 \\
79,7 \\
70,5 \\
78,4 \\
73,5 \\
62,7 \\
51,2 \\
75,2 \\
85,9 \\
- \\
- \\
59,8 \\
- \\
- \\
- \\
-\end{array}$ & $\begin{array}{c}\text { Sand } \\
" \\
" \\
" \\
" \\
" \\
" \\
\text { - } \\
\text { Lehm. Sand } \\
- \\
- \\
- \\
-\end{array}$ \\
\hline
\end{tabular}


1 Ex.: 338 (Deutsche Ems, querab Bentersiel). Das Wasser ist an dieser Stelle oligohalin.

\section{Gammarus salinus Spooner}

Diese Art, die seine Hauptverbreitung in der mesohalinen Zone hat, ist im Dollart-Gebiete erbeutet worden (Mesohalinikum) und in der Ems (von dem mesohalinen Bereich bei Ditzum bis sehr weit stromaufwärts ins süße Teil).

Dollart: 190b (1) (Reiderplate).

$434(\infty)$ (Kerkeriet, aus angeschwemmten Torfschollen).

Schanskerdiep, bei Tonne 1, Kurre, 5-6 m tief, 17 Sept. 1956 (1).

Ems: 332 a (1) (querab Ditzum).

334 a (1) (querab Oldersum).

336 a (2) (querab Kritzum).

337 a (1) (querab Jemgum).

338 (9) (bei Hüttermoor).

339 a (1) (querab Spitland).

$339 \mathrm{~d}(1)$ (bei Eisenbahnbrücke in der Nähe von Weener).

$339 \mathrm{~h}$ (1) (querab Linesch).

Gammarus oceanicus Segerstråle

Diese Art lebt in Wasser mit höherem Salzgehalt als die beiden vorgehenden Arten. Sie liegt von 3 Stationen vor, 1 in der polyhalinen Zone, 2 in der marinen Zone des Estuarium:

Ostlich von Del,;zijl: 352 (1).

Sparregat: 1 Ex. an der Niedrigwasserlinie, 6 Ex. zwischen Grünalgen gesammelt, 19 Sept. 1956.

Familie Talitridae

Hyale spec.

Bei der Station 347 (Östlich von Delfzijl) wurde ein Ex. einer Hyale-Art erbeutet, das sich nicht ohne Weiteres als der an unseren Küsten so allgemeine $H$. nilssoni (Rathke) bestimmen läßt.

Familie Photidae

Microprotopus maculatus Norman

311 (4).

4 Exemplare in Probe 311, mit dem Bodengreifer nördlich der Hund Plate, gefangen.

\section{Familie Corophiidae}

Diese Familie ist mit 4 Arten, wovon 2 im Gebiet zahlreich, und 2 im Gebiet selten, vertreten. Wir werden anfangen die Stationsliste und andere Sammeldaten zu geben, und schließlich die Ókologie der Corophium-Arten betrachten.
Corophium arenarium Crawf.

Das Weibchen von arenarium unterscheidet sich vom Weibchen von volutator in der Abwesenheit eines terminalen Zahns an Antenna 2, Glied 4. Dieses Merkmal ist aber, nach Crawrord, 1937, variabel. Obwohl ich selber viele Hunderte Exemplare untersucht habe und nie solche Abweichungen gefunden habe, liegt noch ein weiteres, sehr befriedigendes Unterschiedsmerkmal vor. Wie ich schon im Jahre 1952 hervorgehoben habe, tragen die 1. Uropoden in arenarium immer eine Anzahl Haare am Außenrand, proximal der Stachelreihe. Solche Haare fehlen in volutator völlig. Dieses Merkmal reicht aus um Männchen, Weibchen und Jungtiere von arenariım zu identifizieren.

Coroph. arenarium ist an den nachfolgenden Stationen im Gebiete gesammelt worden:

127 (4 Juv.); 133 (1); 134 (1); 135 (1 Juv.); 165A (46); 166 (58); 167 (2); 170 (1); 172 (5); 173 (4); 174 (8); 170 (2); 179 (1); 180 (216); 188 (1); 258 (4); 259 (1); 260 (1); 268 (1); 269 (2); 276 (5); 277 (1); 278 (7); 288 (8); 289 (1 Juv.); 394 (2); 396 (2); 398 (14); 399 (21); 400 (12); 401 (35); 410 (20); 438 (3); 439 (5); 440 (6); 441 (11); 442 (3); 443 (3); 444 (4); 445 (3).

\section{Corophium sextoni Crawf.}

402 (1).

Eine marine Corophium, bisher nur einmal in den Niederlanden beobachtet worden (bei IJmuiden; STOCK, 1952). Ein weiteres Exemplar wurde in einer Bodenprobe der Uithuizer Watten gefunden (Stat. 402), wo es im Sande folgender Zusammenstellung lebte:

$$
\begin{array}{rr}
\text { Schlamm }(<16 \mu) & 1.1 \% \\
16-50 \mu & 2.5 \% \\
50-75 \mu & 11.1 \% \\
75-105 \mu & 43.3 \% \\
105-150 \mu & 36.2 \% \\
150-210 \mu & 5.8 \% \\
\cline { 2 - 2 } & 100.0 \%
\end{array}
$$

Corophium lacustre Vanh.

Diese oligohaline und mesohaline Art ist nur an einer Stelle, nämlich Station 212, in der Ems querab Ditzum, zusammen mit Bathyporeia pilosa und $B$. elegans, gefunden worden.

\section{Corophium volutator (Pall.)}

Diese Art ist die allgemeinste Amphipode der Watten. Sie ist an den nachfolgenden Stellen, bisweilen eben massenhaft, angetroffen worden: 
114 (2); 115 (2); 117 (1 Juv.); 127 (9); 131 (26); 132 (25); 133 (11); 134 (18); 135 (46); $136(52) ; 137(69)$; 138 (313); 139 (181); 140 (153); 140A (198); 141 (107); 142 (247); 143 (186); 144 (146); 145 (187); 146 (73); 147 (183); 148 (26); 149 (65); 154 (17); 159 (4); $164(4) ; 165$ (verloren gegangen); 165 A (6 Juv.); 166 (6); 167 (34); 168 (1); 169 (1); 170 (5); 172 (5); 173 (361); 174 (400); 175 (325); $176(483)$; 177 (423); 178 (274); 179 (3); 180 (127); 183 (275); 183A (257); 184 (446); 185 (304); $186(360) ; 187$ (73); 188 (454); 189 (450); 190 (726); $190 \mathrm{~A} \mathrm{(3);}$ 190C (4); 191 (577); 192 (827); 194 (900); 195 (332);
196 (712); 201 (571); 205 (656); 206 (415); 227 (24); 232 (546); $233(530) ; 234$ ( \pm 800$) ; 235$ ( \pm 600$) ; 236$ (269); 237 (372); 238 (439); 239 (252); 240 (108); 241 (2 Juv.); 242 (10); 245 (9); 254 (1); 256 (1 ?); 279 (1); 287 (1); 311 (3 Juv.); 322 (1); 343 (1); 344 (2); 347 (1 Juv.); 349 (5); 350 (3); 361 A (1); 395 (1); 397 (1 Juv.); 400 (28 Juv., 1 Ad.); 401 (4 Juv., 1 Ad.); 410 (4); 416 (1); 417 (2); 419 (1); 422 (57 Ad., 32 Juv.); 423 (49 Ad., 19 Juv.); 424 (5); 425 (70 Ad., 33 Juv.); 426 (119); 427 (3); 429 (51 Ad., 64 Juv.); 434 (2); 464 (91); 465 (60); 466 (23); 467 (14).

\section{OKOLOGIE DER COROPHIUM ARTEN}

Das ökologische Verhalten der Corophium-Arten ist offenbar sehr kompliziert. Wenn wir absehen von Cor. sextoni und Cor. lacustre, welche je nur an einer Stelle gefunden worden sild, sı) bleiben die zwei im Gebiete recht häufig auftretenden Arten Cor. volutator und Cor. arenarium übrig

Die Fazies die von dijesen Arten bewohnt wird, ist abhängig von Faktoren vieler Art:

1) Salinität (beziehungsweise Chlorinität) des umgebenden Wassers;

2) Die Makrostruktur des Bodens (Anwesenheit von Steinen oder Schalen);

3) Die Mikrostruktur des Bodens, insonderheit das Korngrößespektrum;

4) Die Konkurrenz;

5) Die Strömungsgeschwindigk kit;

6) Das Niveau des Standortes bezüglich der Niedrigwasserlinie.

Die Punkte 2, 4, 5, und 6 können kurzgefaßt abgehandelt werden.

ad 2) Die Corophium-Arten meiden Stellen wo die Homogenität des Bodens zerstört wird durch die Anwesenheit vieler Schalen oder Steine.

ad 4) An Stellen wo beide Arten, volutator und arenarium, zusammen vorkommen, wird es volutator häufig gelingen die andere Art zu verdringen. Offenbar nämlich ist volutator im Stande in viel dichteren Populationen standzuhalten als arenarium. Nur an 2 Stationen (Nr. 166 und 180) kamen mehr als 50 Exemplare von arenarium auf der Probefläche vor. Dagegen sind nicht weniger als 62 Stationen bekannt an denen mehr als 50 Exemplare von volutator auf der Probefläche lebten. Die Anzahl kann eben bis etwa 1000 Exemplare zunehmen. Die Fähigkeit massenhaften Auftretens sieht man wohl nur bei Cor. volutator. ad 5) Aus der Tatsache daß die Corophium-Arten nicht in den Prielen leben, scheint man schliefen zu können, daß sie die großen Stromgeschwindigkeiten welche dort bisweilen auftreten, nicht ertragen können. Ubbrigens ist es auch möglich, daß die zu starke Schwankung der Salinität bei Hochund Niedrigwasser die Besiedlung der Priele verhindert.

ad 6) Obwohl die zur Verfügung stehenden Daten wenig zahlreich sind, scheinen sie doch darauf hinzuweisen, daß volutator auch die höheren Teile der Platen bewohnt, weil arenarium sich mehr auf die niedrigen Teilen beschränkt.

Auf die übrigen Faktoren, Salinität und Korngröße soll etwas näher eingegangen werden.

Bezüglich der Salinität kann wohl gesagt werden, daß Cor. arenarium nur die nicht allzu viel versüßten Stellen bewohnt. Sie meidet offenbar den „Sack“ des Dollarts, z.B. Schanskerdiep und Hoogzand. Die Chlorinität des Meereswassers beträgt dort ungefähr $4 \%$ bei niedrigem Wasser und 6-7\% bei hohem Wasser. Anscheinend sind die anderen Faktoren (Korngröße, Konkurrenz, Nahrung) dort nicht ungünstig, sodaß nur der niedrige Salzgehalt an dem Fehlen der Cor. arenarium dort Schuld hat.

Cor. volutator dagegen meidet die marinen Stellen des Gebietes. Sie ist nicht im marinen Bereich der Uithuizer Watten und Rottumeroog gefunden worden. In dem „Sack“ des Dollarts ist sie aber bei einer Chlorinität von weniger als $4 \%$ noch sehr zahlreich.

Die sedimentpetrologische Beschaffenheit des Bodens ist auch sehr wichtig für die Verbreitung von Corophium.

Cor. volutator ist an 115 Stationen im Gebiete angetroffen worden. Die Bodenbeschaffenheit an diesen Stationen ist ziemlich verschieden, aber dennoch sind 
im Vergleich mit den Stationen wo Cor. arenarium lebt, deutliche Unterschiede da.

Erstens ist es auffällig daß Cor. ar. fast ausschließlich Sandböden bewohnt, und in nur 2 Fällen auf lehmigen Sand bestimmt worden ist. Cor. vol. dagegen ist viel weniger wählerisch. Sie ist, wie gesagt, an 115 Stationen bestimmt worden. Der Boden bestand in 33 dieser Stationen aus Sand, in 9 aus lehmigen Sand, in 18 aus sandigem Lehm, in 9 aus Lehm, und in 1 aus tonigem Lehm (in 45 Fällen ist keine Kornanalyse gemacht worden). Weil also Cor. ar. in $90 \%$ aller Fälle im Sand lebt (und im übrigen $10 \%$ im lehmigen Sand), lebt Cor. vol. nur in $46 \%$ aller Fälle im Sande und im Úbrigen im lehmigen Sand, sandigem Lehm und tonigem Lehm.

Die Abbildungen 4 und 5 geben ebenfalls die Korrelation zwischen der Verbreitung der Coro-
phium-Arten und der Korngröße wieder. In diesen graphischen Darstellungen ist aber nicht die oben angewendete Einteilung in Sand, lehmigen Sand, u.s.w. durchgeführt worden, sondern die Verteilung der Körner in einer Fraktion kleiner als $105 \mu$ und ciner Fraktion größer als $105 \mu$.

Auch hier ergibt sich wieder, daß beide Arten durchschnittlich einer etwas anderen Bodenbeschaffenheit den Vorzug geben. Cor. ar. lebt am liebsten in einem Boden wovon durchschnittlich ungefähr $50 \%$ der Körner kleiner ist als $105 \mu$, und selbstverständlich die andere $50 \%$ größer als $105 \mu$. Dagegen lebt Cor. vol. fast immer in einem Boden der für mehr als $50 \%$ (recht häufig viel mehr, sogar bis $99 \%$ ) aus Körnern kleiner als $105 \mu$ besteht.

Die eben genannten Unterschiede der Präferenz betreffs mehr Grob- und mehr Feinkörnigeren-Boden

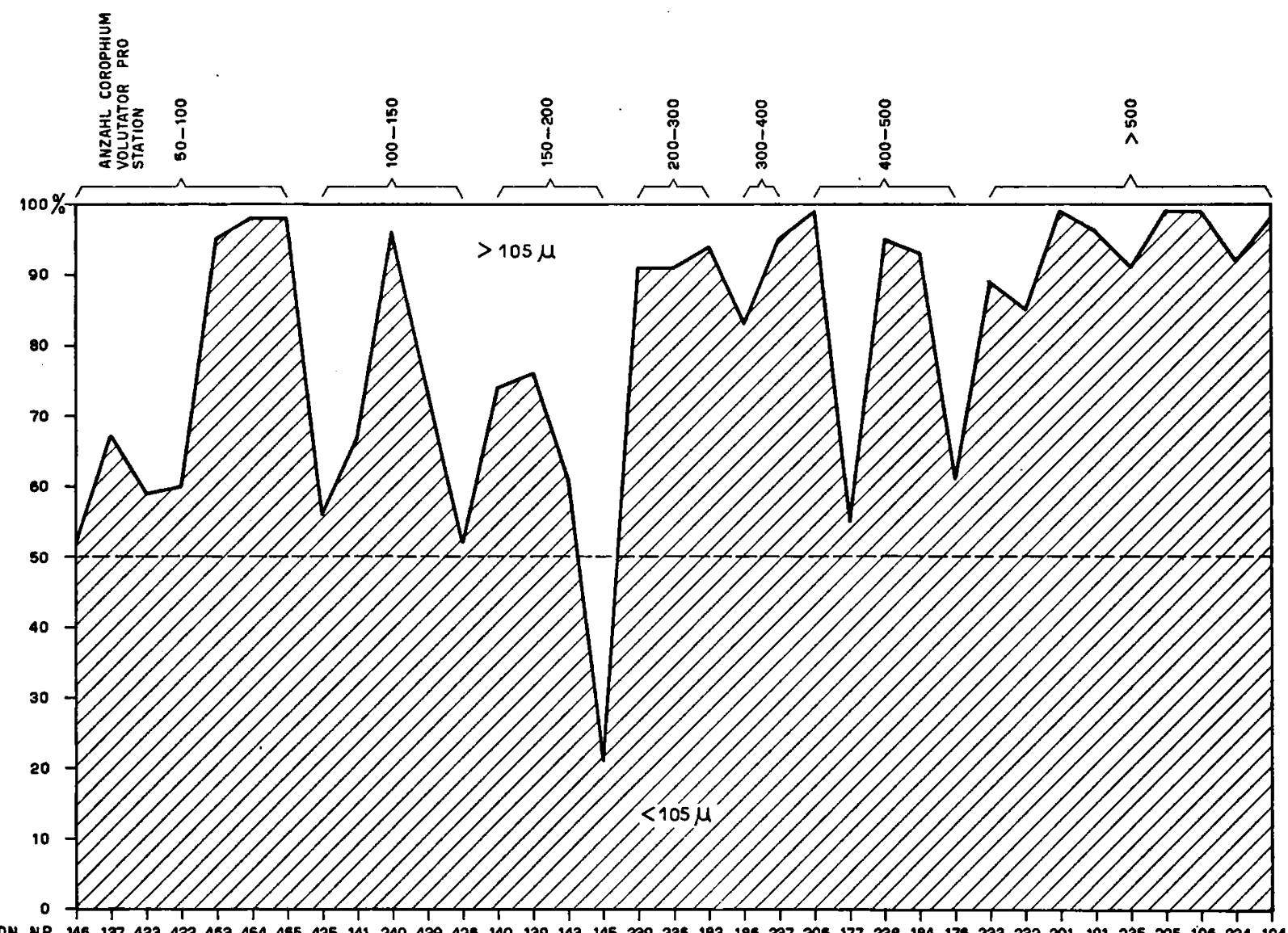

Fig. 4. - Die Bodenbeschaffenheit an 35 Stationen, wo nur Corophium volutator auftrat, und zwar in mehr als 50 Stück pro Probe. An der Apsis, unten, sind die Stationsnummern dargestellt; oben, die Anzahl Exemplare pro Station. An der Ordinat ist der Prozentsatz der Körner größer, beziehungsweise kleiner, als $105 \mu$ dargestellt. 


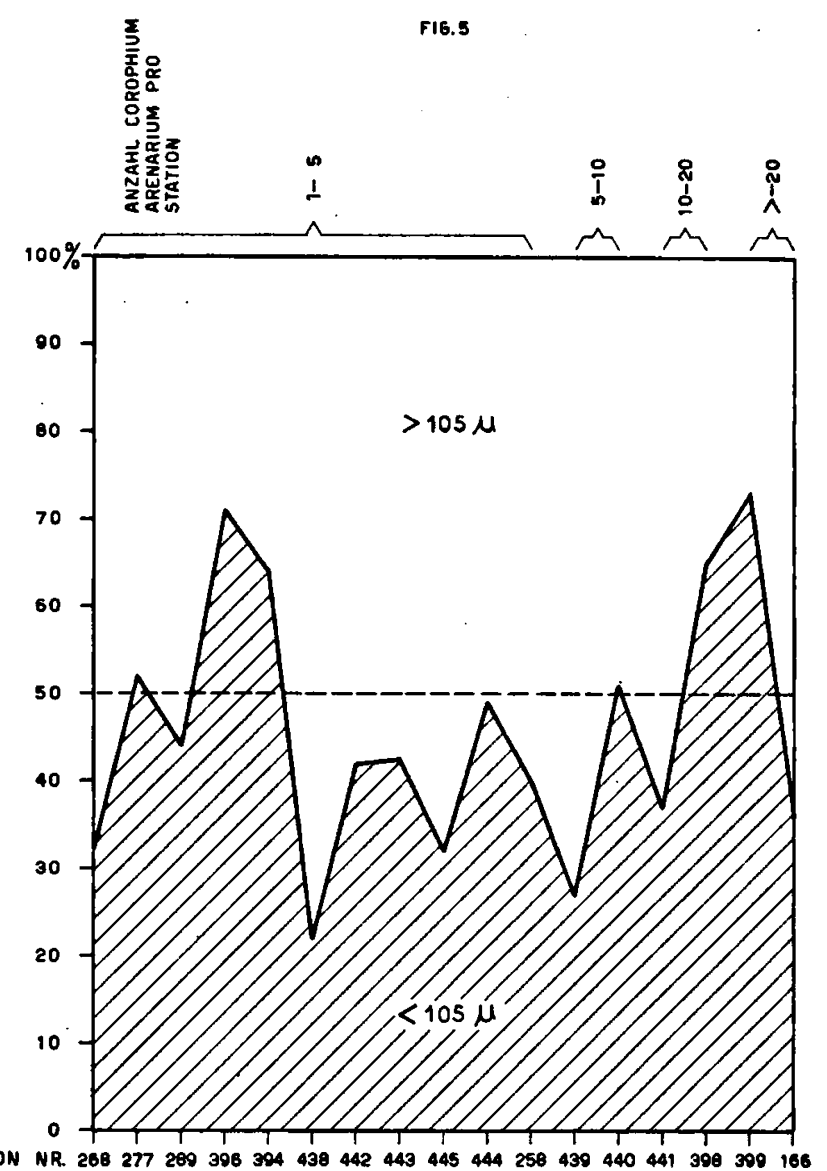

Fig. 5. - Die Bodenbeschaffenheit an den Stationen, wo nur Corophium arenarium auftrat. Erklärung wie in Abb. 4

ist statistisch geprüft worden, und es ergab sich daß die $105 \mu$ Grenze, wie oben auseinandergesetzt, in hohem Maße signifikant war.

Alles zusammenfassend ergibt sich das folgende Bild der ökologischen Verbreitung von Corophium volutator und von $C$. arenarium:

Beide Arten meiden: die Prielen mit stärkerem Strom; die Stellen mit Schalen im Boden. Cor. ar. meidet auch die höheren Teilen der Platen. Für die allgemeine Verbreitung ist erstens die Salinität des umgebenden Wassers verantwortlich. So findet man Cor. vol. gar nicht in dem marinen Teil des Estuariums (z.B. Rottumeroog, Uithuizer Watten) weil dagegen Cor. ar. den mehr brackischen Teil des Gebietes meidet (zB. dem Sack des Dollarts, wie Schanskerdiep und Hoogzand). Innerhalb der von der Salinität bedingten Verbreitungsmöglichkeit, ist die Zusammensetzung des Bodens von vorwiegender Wichtig- keit. So ist Cor. ar. fast ganz an Sandböden gebunden, worin der Anteil der Körner unter $105 \mu$ etwa $50 \%$ beträgt. Cor. vol. ist plastischer: sie nimmt sowohl Sand als auch lehmiger Sand, sandiger Lehm und Lehm vor lieb. Der Anteil der kleineren Körner, also unter $105 \mu$, ist gewöhnlich an diesen Stellen mehr als $50 \%$, und öfters sogar bis $99 \%$.

\section{IsOPODA}

Eurydyce pulchra Leach

3 Ex. -363.

2 Ex. - Ra, auf Licht, 20 Sept. 1956.

Idotea balthica (Pall.)

1 Ex. - Ra, auf Licht, 20 Sept. 1956.

Jaera albifrons Leach

Ca. 10 Ex. Rottumeroog, 12 Aug. 1954. Muschelbank auf den Watten. 


\author{
ANISOPODA \\ Tanaissus lilljeborgi (Stebbing) \\ 1 Ex. - 318. \\ 1 Ex. - 356. \\ 2 Ex. -360. \\ 1 Ex. -361. \\ 1 Ex. - 365.
}

Diese kleine Art scheint auf sandigen Watten in der Wattensee nicht selten zu sein. Beim Sieben der Bodenproben schlüpfen die Tiere aber vielfach durch die Maschen des Siebes.

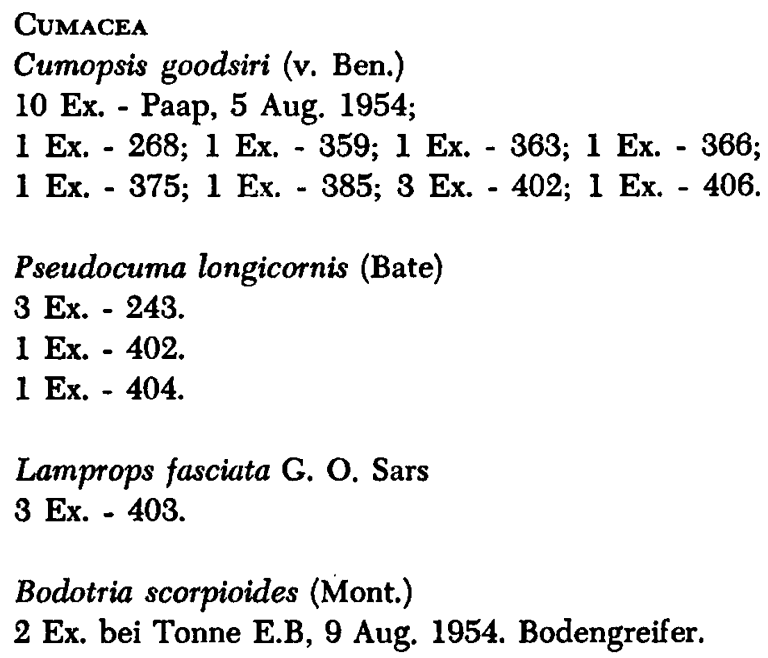

Balaniden kommen allerorts auf festem Substrat vor. Die Arten sind denn auch viel allgemeiner als sich aus dieser kurzen Liste ergibt.

Sacculina carcini Thompson

Nicht selten auf der Krabbe Carcinus schmarotzend, besonders an meeresnahen Stellen.

\section{Mysidacea}

Neomysis integer (Leach)

245; 260; bei 463 (je 1 Stück); 259 (100 Ex.); 212 (42 Ex.).

Mesopodopsis slabberi (van Beneden)

1 Ex. - 183a; 1 Ex. - 190C; 2 Ex. - 239; 2 Ex. - 241;

1 Ex. - 244; 2 Ex. - 254; 1 Ex. - 259-262; 1 Ex. 393; 1 Ex. - 412; 4 Ex. - 436; 3 Ex. - 445; 1 Ex. 447; 1 Ex. - 465.

Paap, Rand der Plate: 1 Ex. 5 Aug. 1954.

Praunus flexuosus (O.F.M.)

1 Ex. - Rottumeroog (12 Aug. 1954).

1 Ex. - Paap, auf den Watten (5 Aug. 1954).

DECAPODA

Carcinus moenas (L.)

Zahlreich im ganzen Gebiete.

Crangon crangon (L.)

An sandigen Stellen im ganzen Gebiete zahlreich.

Eriocheir chinensis M. Edw.

Die Wollhandkrabbe ist nur in 2 jungen Exemplaren beobachtet worden. Nieuwe Statenzijl, Außenseite der Schleuse unter Steinen, 19 Sept. 1956.

\section{PYCNOGONIDA}

Phoxichilidium femoratum (Rathke)

1 Larve - Paap, auf Hydroiden. 5 Aug. 1954.

\section{ZUSAMMENFASSENDE BETRACHTUNGEN}

Das ganze Gebiet, von Rottumeroog im Norden bis zum Zusammenfluß von Ems und Leda im Südosten, steht unter dem Einfluß von zwei sich widerstrebender Komponenten: einerseits das zum Meer abströmende süße Fluß- und Polderwasser, andrerseits das aus dem Norden vordrängende salzige Meerwasser. Die Fauna des Gebietes steht im Zeichen dieses Kampfes zwischen salzigem und süßem Wasser.
Weitaus die meisten Bewohner sind stark euryhaline Arten, Arten also, die große Schwankungen im Salzgehalt ertragen können. Mehr als eine Art kommt im ganzen Gebiet vor. Beispiele sind die Copepoden Euterpina acutifrons und Platychelipus littoralis. Dennoch kann man das Gebiet in fünf mehr oder weniger deutliche Zonen aufgliedern, jede Zone mit einer eigenen faunistischen Zusammensetzung. Nur 2 
dieser Zonen sind in positiver Weise gekennzeichnet, nämlich durch den Besitz einer Fauna mit einer Anzahl kennzeichnender Arten, die nicht außerhalb ihrer eigenen Zone vorkommen. Die übrigen Zonen haben präferente Arten, die zwar in dieser Zone ihre optimale Verbreitung haben, jedoch auch außerhalb dieser Zone vorkommen. Außerdem sind letztgenannte Zonen besonders gekennzeichnet durch das Fehlen bestimmter Arten.

Gut gekennzeichnet ist erstens die marine Zone. Sie gehört eigentlich nur geographisch zum EmsEstuarium, faunistisch gehört das ganze Gebiet zum Wattenmeer. Das Watt unter Rottumeroog und die Uithuizer Watten gehören dazu: die Grenze liegt ungefähr bei der Hond Plate. Kennzeichnend sind der Wurm Echiurus echiurus, der Seestern Asterias, die Copepoden Leptinogaster, Macrochiron, Modiolicola, Mytilicola, Canuella furcigera, Pseudobradya beduina, Tisbe furcata, die Amphipoden Urothoe, Bathyporeia pelagica, Corophium sextoni, die Anisopode Tanaissus, u.s.w.

Die zweite, gut gekennzeichnete Zone ist die Süßwasserzone der Ems, von dem Zusammenfluß mit der
Leda stromaufwärts. Die Süßwassercopepoden des Genus Cyclops und eine ganze Reihe von Cladocera kennzeichnen diese Zone.

Die polyhaline Zone, die Umgebung von Delfzijl und der Paap Plate, ist die äußerste Grenze, bis wohin noch marine-polyhaline Arten, wie Paracalanus, Asellopsis, Harpacticus-Arten, Haustorius, Bathyporeia sarsi, vordringen können. Für eine Anzahl Brackwasserorganismen, wie Corophium volutator und Nannopus palustris, wird hier der Maximalwert des Salzgehaltes erreicht.

Das übrige Gebiet gehört zur mesohalinen Zone, ziemlich deutlich sind jedoch die südöstliche Gegend (der "Sack“) des Dollarts (Schanskerdiep, Hoogzand) und die Ems-Mündung vom übrigen Teil getrennt. Diese Gebiete mit ihrem niedrigen Cl-Gehalt $(<$ $5,5 \%$ ) gehören der $\alpha$-mesohalinen Zone an. In der Fauna fällt u.a. das Fehlen von Corophium arenarium, sowie die Häufigkeit von Gammarus salinus, auf. In der $\beta$-mesohalinen Zone $(\mathrm{Cl}>5,5 \%$ ), wozu u.a. Kerkeriet- und Reider Plate gehören, tritt Corophium arenarium dagegen wohl auf.

\section{SCHRIFTTUM}

Bocquet, C. et J. H. STock (1958) - Copépodes parasites d'Invertébrés des Côtes de la Manche. IV. Sur les trois genres synonymes de Copépodes Cyclopoïdes, Leptinogaster Pelseneer, Strongylopleura Pelseneer et Myocheres Wilson. Archs. Zool. Exp. Gén., 96 (N. \& R. 2), S. 71-89.

Crawford, G. I. (1937) - A review of the amphipod genus Corophium, with notes on the British species. Journ. Mar. Biol. Ass. U.K., 21 (N.S.), S. 589-630.

Gurney, R. (1931) - British Fresh-water Copepoda, vol. I. Ray Soc. Monogr., 88, lii + $238 \mathrm{~S}$.

Hansen, H. J. (1897) - The Choniostomatidae, a family of Copepoda parasitic on Crustacea Malacostraca. København, $206 \mathrm{~S}$.

Lang, K. (1948) - Monographie der Harpacticiden, I, II. Lund, 1683 pp.
Pelseneer, P. (1929) - Copépodes parasites de Mollusques. Ann. Soc. Roy. Zool. Belgique, 59, S. 33-49.

Redexe, H. C. (1935) - Acartia (Acanthacartia) tonsa Dana ein neuer Copepode des Niederländischen Brackwassers. Archs. Néerl. Zool., 1 (3), S. 315-329.

Stock, J. H. (1952) - Some notes on the taxonomy, the distribution and the ecology of four species of the Amphipod genus Corophium. Beaufortia, 2 (21), S. 1-10.

Stock, J. H. (1957) - Somes notes on the genus Macrochiron Brady, 1872. Ann. Mag. Nat. Hist. (12), 10, S. 378-382.

Vos, A. P. C. de en H. C. Redeke (1941) - Bijdragen tot de kennis van de mikrofauna der Groninger en Uithuizer Wadden. Publ. Hydrobiol. Club Amsterdam, 3, S. 1-31. 